\title{
Influence of External Coagulant Water Types on the Performances of PES Ultrafiltration Membranes
}

\author{
Jing He, Lingyun Ji and Baoli Shi*
}

\author{
Polymer Membrane Laboratory, College of Science, Northeast Forestry University, Harbin, Heilongjiang, \\ China
}

\begin{abstract}
In order to research the influence of cluster structure on the performances of membranes prepared through immersion precipitation phase inversion technique when water was used as external coagulant, in this paper, two types of water were prepared from distilled water and were used as external coagulant at room temperature. One type (water I) was prepared from boiled distilled water and another type (water II) was prepared from ice. Six polyethersulfone membranes were prepared by using polyvinylpyrrolidone as additive. Pure water flux, permeation flux of BSA solution and rejection to bovine serum albumin of the membranes were investigated. It was found that according to the statistically experimental results, the membranes prepared in water II always had larger pure water flux, permeation flux of BSA solution, rejection and swelling ratio than the membranes prepared in water I. Consequently, the types of water external coagulant had some influences on the separation performances of the prepared membranes but had no effects on the surface chemical groups. However, the present morphology characterization technology, such as SEM couldn't detect the tiny difference in the pore structures.
\end{abstract}

Keywords: Polyethersulfone, ultrafiltration, water clusters, phase inversion.

\section{INTRODUCTION}

Immersion precipitation phase inversion technique is a common method for the preparation of polymer separation membranes, such as ultrafiltration membranes and nanofiltration membranes [1]. For this membrane preparation technique, the nascent polymer membranes (hollow fiber or flat sheet membranes) need to be dipped in a liquid external coagulant to take place the phase inversion process. Generally, water is used as the external coagulant because water is a very cheap and safe liquid.

Although water is the common liquid, it is the strangest matter on our earth [2]. Compared with other small molecules, water has lots of strange performances. Its strange behaviors come from its unusual structures. Until now, more than forty-six hypothetical models have been developed to explain its structures [2]. However, one common concept has been widely accepted that is liquid water is not homogeneous at nanoscopic level. Different water clusters exist within liquid water. Some clusters are low density which is similar with the density of hexagonal ice: four water molecules come together to form a bicyclo-octamers, and some clusters may be higher density as shown in Figure 1.

The relative concentrations of different clusters within liquid water can be changed through heating. For

*Address corresponding to this author at the Polymer Membrane Laboratory, College of Science, Northeast Forestry University, Harbin, Heilongjiang, China; Tel: +86 4518219 2327; Fax: +86 45182192327 ;

E-mail:shi_baoli@yahoo.com liquid water at room temperature, when it is heated to boil, the larger clusters can be broken to smaller clusters. After it is cooled to the initial room temperature, its cluster status will be changed comparing with its initial status, and some physical properties will also change.

As our best knowledge, there is no research report on if the water types or water cluster structures will influence the membrane performances when water is used as external coagulant during immersion precipitation phase inversion process. In this paper, we prepared two types of water and used them as external coagulant to research the above question. We chose polyethersulfone (PES) as the membrane material to prepare ultrafiltration flat sheet membranes by adding polyvinylpyrrolidone (PVP K30). The morphology, water flux and rejection to bovine serum albumin (BSA) of the membranes were investigated to validate the influence of water types. We think that the basic research work will be benefit to the preparation of ultrafiltration or nanofiltration membranes.

\section{EXPERIMENTAL}

\subsection{Materials}

PES (Ultrason E 6020P) was purchased from BASF Co., Germany. PVP (K30) was purchased from Fluka Chemika, Switzerland. Bovine Serum Albumin (BSA) $\left(M_{w}\right.$ was 68,000) was purchased from BeiJing AoBoXing Bio-tech Co., Ltd., China. N-methyl-2-pyrrolidone (NMP) was used as solvent which was purchased from Tianjin 


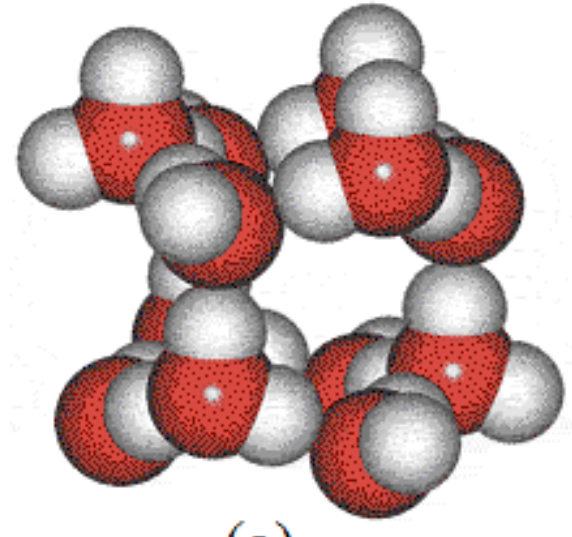

(a)

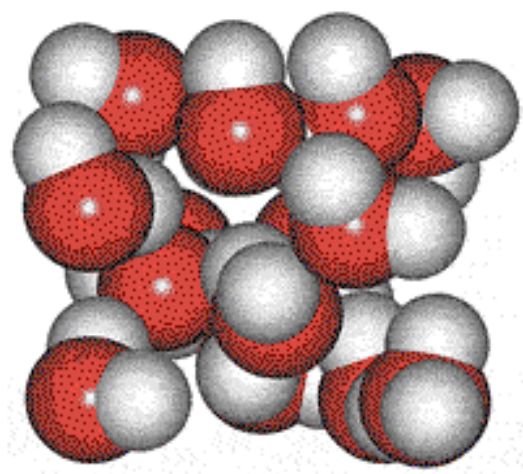

(b)

Figure 1: Schematic cluster structures of low-density ice-like water (a) and high-density liquid water (b) [2].

Kermel Chemical Reagents Development Centre, China.

\subsection{Preparation of UF Membranes}

\subsubsection{Preparation of External Coagulant Water}

Two types of water were prepared from distilled water and were used as external coagulant at room temperature, $20^{\circ} \mathrm{C}$. One type (named as water I) was prepared from boiled distilled water: distilled water was first heated to boil and was kept boiling for 25 minutes, and then it was naturally cooled to room temperature during one night. Another type (named as water II) was prepared from ice: distilled water was first frozen to form ice in a refrigerator at $-24^{\circ} \mathrm{C}$, and then the ice naturally melted at room temperature during one night.

\subsubsection{Preparation of PES UF Membranes}

PES ultrafiltration membranes were prepared by the common immersion precipitation phase inversion method. PES was first dried at $100^{\circ} \mathrm{C}$ for at least $24 \mathrm{~h}$ before use. Homogeneous casting solution was prepared by dissolving PES and PVP in NMP at $50^{\circ} \mathrm{C}$. The casting solution was cast on non-woven fabric support and then was immersed into water bath containing the above waters and kept in the water bath for at least $48 \mathrm{~h}$ until most of the solvent was removed. The thickness of the prepared membranes was 200$400 \mu \mathrm{m}$.

\subsection{Characterization of the Membranes}

\subsubsection{Characterization of Top Surface and Cross Section Morphology of the Membranes by SEM}

The top surface and cross section morphology of the prepared membranes was examined by a scanning electron microscope (FEI Quanta 200, USA). The samples were frozen in liquid nitrogen and were fractured. After sputtering with gold, the top surface and cross-section of the membranes was viewed with the SEM.

\subsubsection{Characterization of Membrane Surface by ATR-FTIR Spectroscopy}

The surface of the PES membranes were analyzed by attenuated total reflectance Fourier transform infrared spectroscopy (ATR-FTIR) technique, which was performed on a Nicolet FT-IR 360 Spectrometer. The surface of the membranes was in contact with a ZnSe crystal with a $45^{\circ}$ angle of incidence. Absorbance spectra were obtained in the region of $4000-500 \mathrm{~cm}^{-1}$ with a resolution of $4 \mathrm{~cm}^{-1} .64$ scans were averaged in order to obtain clear absorption spectra of each sample.

\subsubsection{Swelling Ratio of the Membranes in Water}

Swelling ratio of the membranes in distilled water was measured at room temperature. First, the prepared membranes were dipped in ethanol for $10 \mathrm{~h}$ and then were dried naturally. After weighting their mass, they were dipped in distilled water at room temperature for $24 \mathrm{~h}$. When they were taken from the water, they were wiped with filter paper quickly and weighted. The swelling ratio $\left(S_{w}\right)$ was calculated with the following equation:

$S_{w}=\frac{m_{w}-m_{0}}{m_{0}}$

where $m_{w}$ was the mass of membrane containing water, $m_{0}$ was the mass of dry membrane.

\subsection{Ultrafiltration Experiment}

Ultrafiltration experiment was carried out by using a self made equipment as shown in Figure 2 at room 


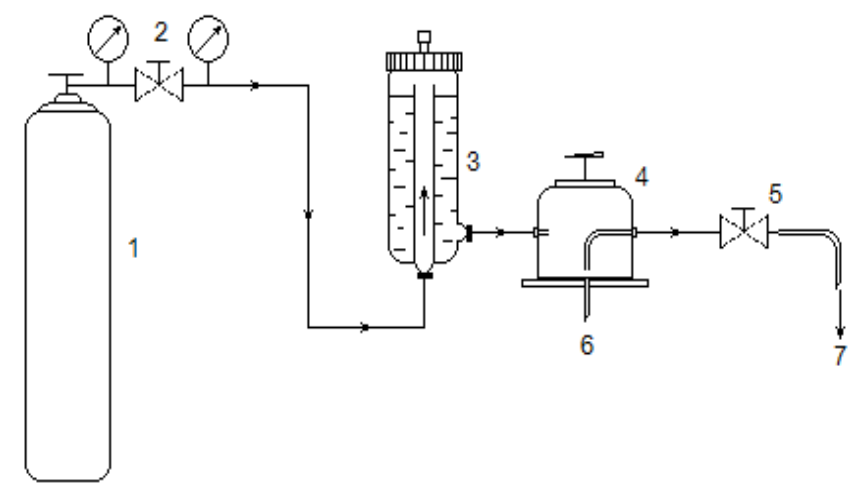

Figure 2: Schematic diagram of UF separation experimental equipment. 1.Nitrogen gas, 2.valve, 3.feed tank, 4.membrane testing cell, 5.value, 6.permeation, 7.concentrated solution.

temperature. Pure water flux was measured through dead end filtration method and concentration separation experiment was operated through cross flow filtration method. The effective area of the membrane was $7 \mathrm{~cm}^{2}$. The pressure in the feed side was maintained at $100 \mathrm{kPa}$ with nitrogen gas. The pure water flux, $J_{w}$ of the membrane was calculated by the following equation:

$J_{w}=\frac{V}{A \Delta t}$

where $V$ was the volume of permeation water. $A$ was the membrane area and $\Delta t$ was the permeation time. For the concentration separation experiment, two liters of BSA aqueous solution $(1 \mathrm{mg} / \mathrm{mL})$ was used as the feed solution for the rejection test. The BSA concentration of permeation was measured by a UV-vis spectrophotometer (T6, Beijing Purkinje General Instrument, China). The rejection $(R)$ was calculated by the following equation:

$R=\left(1-\frac{C_{p}}{C_{f}}\right) \times 100$ where $C_{p}$ and $C_{f}$ were the concentrations of BSA in the permeation and feed side, respectively.

\section{RESULTS AND DISCUSSION}

Table 1 lists the average pure water flux, the average permeation flux of BSA solution, the average concentration of BSA in permeation, the average rejection to BSA and swell ratio of three groups of PES ultrafiltration membranes. According to the listed data, for any type of the membranes, the average pure water flux is almost two times of the average permeation flux of BSA solution. For any group of the membranes, from a statistical viewpoint, the membranes prepared in water II always have larger pure water flux, permeation flux of BSA solution, rejection and swelling ratio than the membranes prepared in water I. Consequently, the types of water external coagulant have some influences on the prepared membranes.

Figures 3-5 show the cross-section morphology of the membranes and Figure 6 presents the top surface morphology of two membranes in group II. It seems that there is no significant difference in the membrane structure for the two membranes in one group. Figure 7 shows the ATR-FTIR spectroscopy of two membranes in group II. There is no significant difference in the main reflectance peaks. Consequently, the functional groups in the surface of the two membranes were almost same. It also means that the external coagulant water types have no influence on the chemical groups in the membrane surface.

The possible reason for the influence of external coagulant water types on the separation performances of the PES membranes is that the cluster structure of water has an effect on the pore formation. It was found that the melting ice could release low-density water clusters and the clusters could keep their structures for a long time [2]. Consequently, melted ice and boiled

Table 1: Performances of PES Ultrafiltration Membranes

\begin{tabular}{|c|c|c|c|c|c|c|c|c|}
\hline \multirow{2}{*}{ Group } & Number & $\begin{array}{c}\text { Casting solution } \\
\text { composition }\end{array}$ & $\begin{array}{c}\text { External } \\
\text { coagulant }\end{array}$ & $J_{w}\left(\mathbf{L} / \mathbf{m}^{2} \mathbf{h}\right)$ & $J_{p}\left(\mathrm{~L} / \mathbf{m}^{2} \mathbf{h}\right)$ & $\boldsymbol{C}_{p}(\mathbf{m g} / \mathbf{m L})$ & $R(\%)$ & $\boldsymbol{S}_{w}(\%)$ \\
\hline \hline \multirow{2}{*}{ I } & $(1)$ & PES(24\%) & water I & $3.4 \pm 0.5$ & $1.6 \pm 0.3$ & $0.248 \pm 0.088$ & $75.2 \pm 8.8$ & 27.7 \\
\cline { 2 - 9 } & $(2)$ & PES(24\%) & water II & $5.9 \pm 0.8$ & $2.7 \pm 0.4$ & $0.083 \pm 0.035$ & $91.7 \pm 3.5$ & 32.5 \\
\hline \multirow{2}{*}{ II } & $(3)$ & PES(17\%)/PVP(2\%) & water I & $233.6 \pm 11.5$ & $110.5 \pm 5.0$ & $0.050 \pm 0.041$ & $95.0 \pm 4.1$ & 69.4 \\
\cline { 2 - 9 } & $(4)$ & PES(17\%)/PVP(2\%) & water II & $255.6 \pm 10.6$ & $130.7 \pm 6.4$ & $0.039 \pm 0.034$ & $96.1 \pm 3.4$ & 73.4 \\
\hline \multirow{2}{*}{ III } & $(5)$ & PES(17\%)/PVP(12\%) & water I & $203.1 \pm 12.6$ & $95.9 \pm 7.1$ & $0.218 \pm 0.115$ & $78.2 \pm 11.5$ & 76.6 \\
\cline { 2 - 9 } & $(6)$ & PES(17\%)/PVP(12\%) & water II & $236.8 \pm 9.7$ & $120.4 \pm 5.8$ & $0.070 \pm 0.054$ & $93.0 \pm 5.4$ & 77.8 \\
\hline
\end{tabular}

$J_{w}$ : pure water flux; $J_{p}$ : permeation flux of BSA solution; $C_{p}$ : concentration of BSA in permeation; $R$ : rejection to BSA; $S_{w}$ : swelling ratio. 


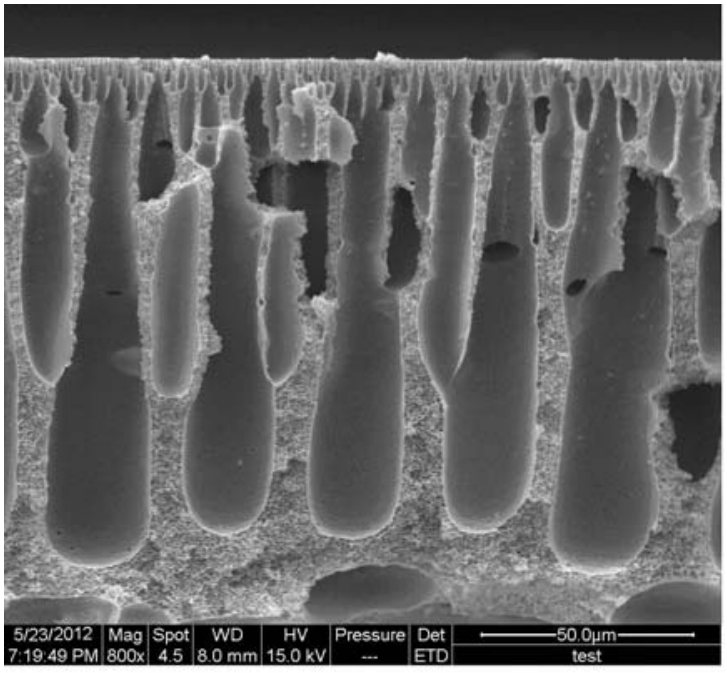

Group I Number (1) - water I

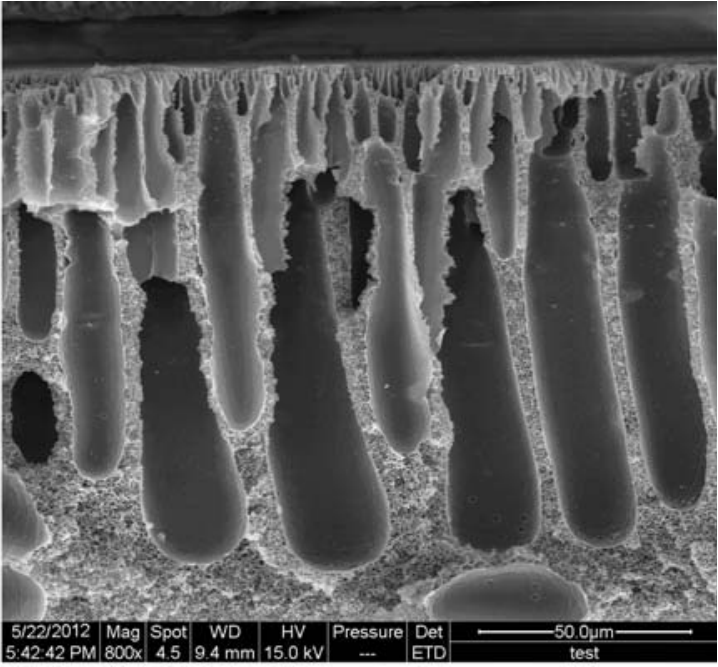

Number (2) - water II

Figure 3: Cross-section morphology of two membranes in group I.

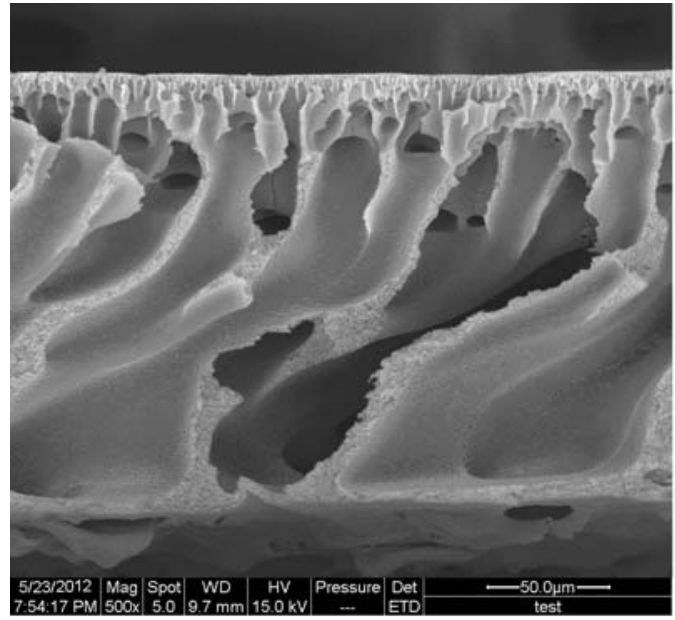

Group II Number (3) - water I

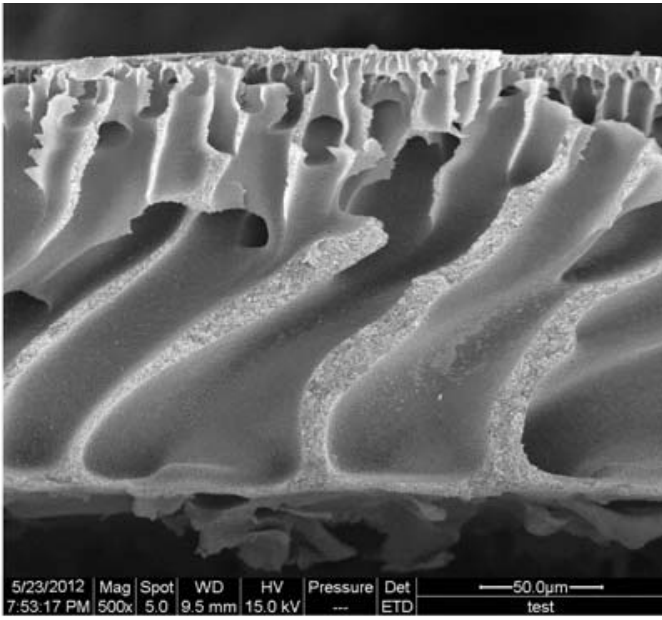

Number (4) - water II

Figure 4: Cross-section morphology of two membranes in group II.

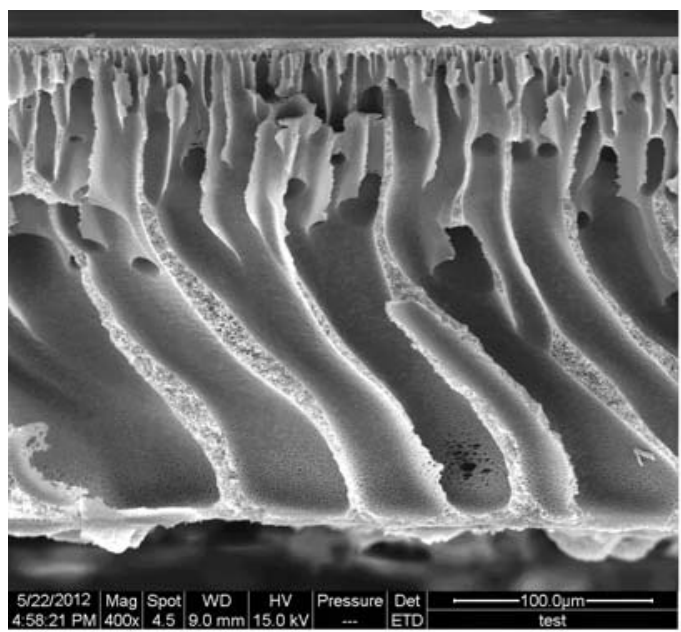

Group III Number (5) - water I

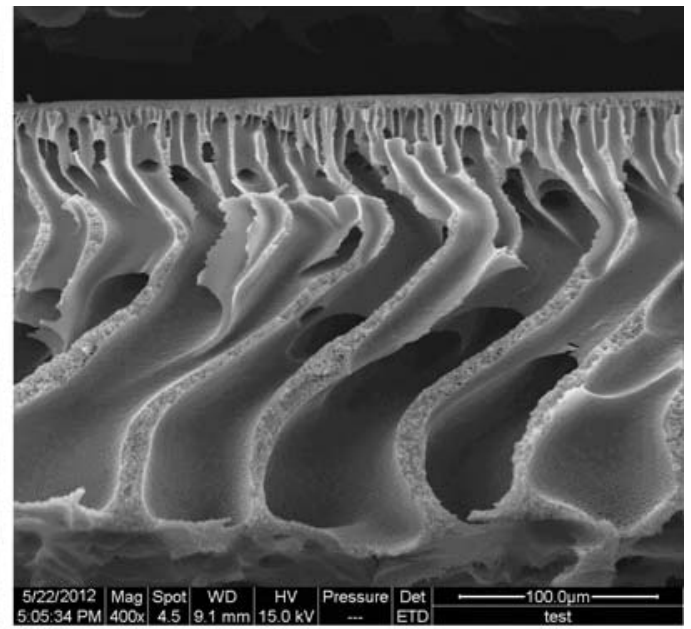

Number (6) - water II

Figure 5: Cross-section morphology of two membranes in group III. 


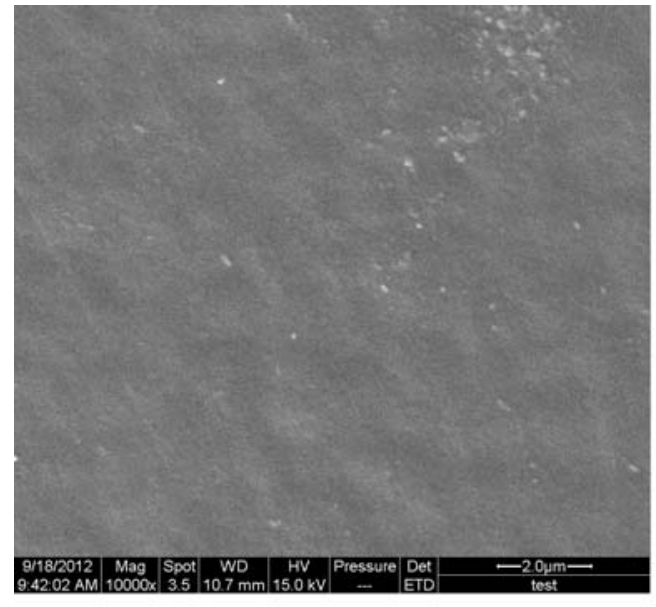

Group II Number (3) - water I

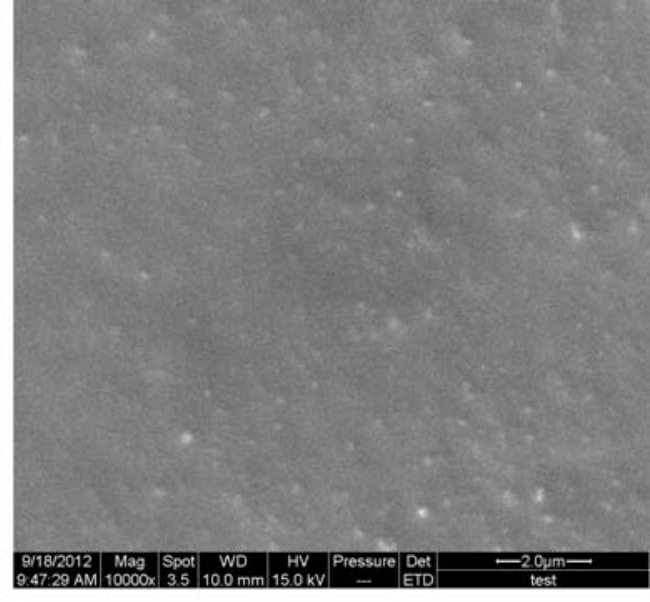

Number (4) - water II

Figure 6: Top surface morphology of two membranes in group II.

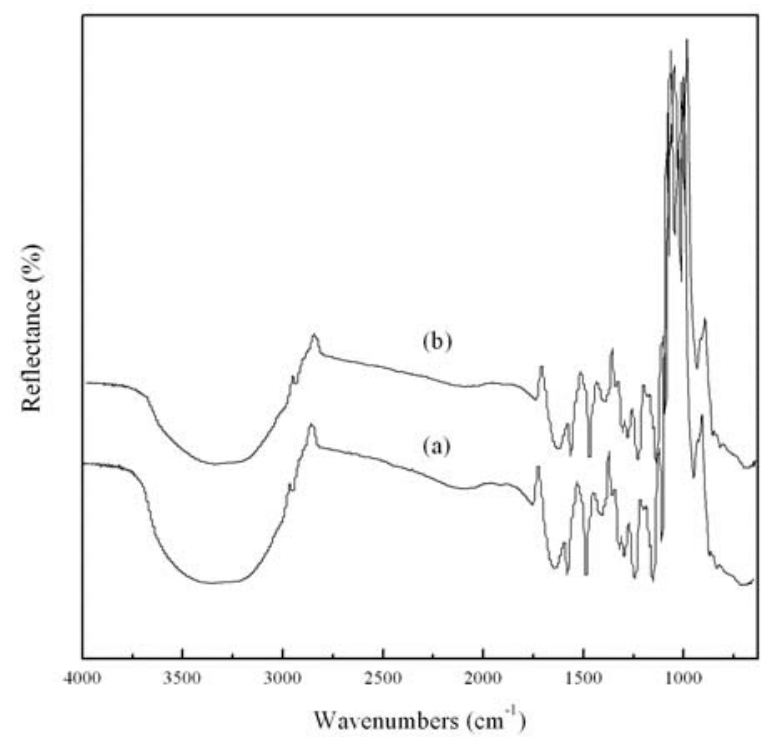

Figure 7: FTIR spectra of top surface of two membranes in group II: (a) number (3) - water I, (b) number (4) - water II.

water would have different cluster structures, and the structures would partially maintain when they reached to the same temperature. This difference in cluster structures would finally influence the membrane structure of the micro-pores. However, the present morphology characterization technology, such as SEM, couldn't detect the tiny difference in the pore structures.

\section{CONCLUSION}

Two types of water were prepared from distilled water and were used as external coagulant at room temperature. One type (water I) was prepared from boiled distilled water and another type (water II) was prepared from ice. The cluster structures of them should be different. It was found that according to the statistically experimental results, the membranes prepared in water II always had larger pure water flux, permeation flux of BSA solution, rejection and swelling ratio than the membranes prepared in water I. Consequently, the types of water external coagulant had some influences on the separation performances of the prepared membranes but had no effects on the surface chemical groups. However, the present morphology characterization technology, such as SEM, couldn't detect the tiny difference in the pore structures.

\section{ACKNOWLEDGEMENTS}

The authors acknowledge the financial support by the Fundamental Research Funds for the Central Universities of China (DL12EB05-03) and National Natural Science Foundation of China (20906010).

\section{REFERENCE}

[1] Baker RW, Ed. Membrane technology and applications. England: Wiley 2004.

[2] Water structure and science. London South Bank University; [updated by Martin Chaplin on 2012 Aug 4; cited 2012 Sep 4]: Available from: http://www.Isbu.ac.uk/water/ 